\title{
OCUPAĈ̃O E RENDA DA MULHER NO NORDESTE: EVIDÊNCIAS A PARTIR DA PNAD 2001 A 2015
}

\author{
Rayla Keyla da Silva ${ }^{1}$ \\ José Antonio Nunes de Souza ${ }^{2}$ \\ Francisco Danilo da Silva Ferreira ${ }^{3}$ \\ Vinicius Rodrigues Vieira Fernandes ${ }^{4}$ \\ Ricardo Vitor Fernandes da Silva ${ }^{5}$
}

RESUMO: O presente estudo tem como objetivo geral analisar a trajetória das mulheres ocupadas na região nordeste. Inicialmente a pesquisa se norteou pela análise da inserção da mulher no mercado de trabalho, e em seguida pela investigação do perfil mais geral da mão de obra feminina ocupada no Nordeste nos anos mais recentes. Metodologicamente, foi realizado um levantamento a partir da Pesquisa Nacional por Amostra Domiciliar (PNAD) do Instituto Brasileiro de Geografia e Estatística (IBGE) no período de 2001 a 2015. De modo geral, os dados mostraram que a participação feminina no mercado de trabalho elevou a renda das mulheres, o que pode ter sido provocado dentre outros fatores pela melhora da escolaridade da mão de obra feminina. Outro ponto relevante foi a observação de um aumento considerável acerca dos domicílios chefiados por mulheres, as quais se destacaram nas atividades de educação, saúde e serviços sociais.

Palavras-Chave: emprego; gênero; ocupação; renda.

ABSTRACT: The present study aims to analyze the trajectory of employed women in the northeast region. Initially, the research was guided by the analysis of the insertion of women in the labor market, and then by the investigation of the more general profile of female labor force employed in the Northeast in more recent years. Methodologically, a survey was carried out from the National Household Sample Survey (PNAD) of the Brazilian Institute of Geography and Statistics (IBGE) in the period from 2001 to 2015 . In general, the data showed that female participation in the labor market increased women's income, which may have been caused, among other factors, by the improvement in the education of female labor. Another relevant point was the observation of a considerable increase in households headed by women, which stood out in the activities of education, health and social services.

Keywords: employment; gender; occupation; income.

\footnotetext{
${ }^{1}$ Economista pela UERN. E-mail: raylakeyla@hotmail.com.

2 Mestrado em economia (UFRN). É especialista em Economia da Saúde (USP). Graduado em Economia (UERN). Professor do departamento de economia (UERN). E-mail: raylakeyla@hotmail.com.

${ }^{3}$ Graduado em Economia (UERN). Mestrado em Economia (UFRN). Professor Assistente da (UERN). Doutorando em Economia (UFPB). E-mail: ffdaniloferreira@gmail.com.

${ }^{4}$ Graduação e Mestrado em Ciências Econômicas (UFRN). Doutorado em Economia (UFU). Atualmente é Professor Adjunto do Departamento de Economia (UFR). E-mail: vinirvf@gmail.com.

${ }^{5}$ MBA em Administração Financeira (FACEX). Graduação em CST em Gestão Financeira (UNP). Professor Universitário na Universidade Potiguar - UNP. E-mail: rvitornt|@gmail.com.
} 


\section{INTRODUÇÃO}

A ampliação da participação feminina no mercado de trabalho tem sido observada nas economias mundiais ao longo das últimas décadas. No brasil, as estatísticas de gênero no mercado de trabalho apontam para um crescimento ao longo do tempo da taxa de ocupação feminina, embora ainda existam algumas disparidades de gênero no mercado de trabalho brasileiro.

As estatísticas recentes de gênero disponibilizadas pela Pesquisa Nacional por Amostragem de Domicílio (PNAD) mostraram que as mulheres dedicaram aos cuidados de pessoas e/ou afazeres domésticos cerca de $73 \%$ a mais de horas do que os homens, sendo que na Região Nordeste esse percentual chega a $80 \%$. Evidenciando que a estrutura domiciliar tem forte influência da decisão de participação no mercado de trabalho.

Em termos de ocupação as mulheres representam $52,4 \%$ da população em idade de trabalhar respondendo por $45,6 \%$ da ocupação no mercado de trabalho no Brasil. Essas evidências têm chamado atenção de pesquisadores que buscaram analisar fatores determinantes da participação feminina e, da mesma forma, das características da mão de obra ocupada.

Barbosa (2014) Explica que a taxa de atividade feminina no brasil ampliou de forma expressiva ao longo dos anos, atribuindo a escolaridade um fator importante nesse processo. Nessa mesma linha Leone e Teixeira (2016) apontaram que o crescimento da participação feminina no mercado de trabalho brasileiro se intensifica na década 1970 como resultado do processo de industrialização de décadas anteriores, continuou crescendo nas décadas seguintes mesmo diante de cenários econômicos desfavoráveis.

Em termos dos determinantes da participação feminina Resende (2016) aponta que a oferta de trabalho das mulheres possui forte influência da escolaridade e da qualificação. Cirino (2011) explica que aspectos ligados a renda familiar e renda do cônjuge afetam a probabilidade da inserção feminina no mercado de trabalho. Ramos et al (2011) coloca a presença de filhos, sobretudo, nas menores faixas etárias como um determinante significativo da participação feminina na atividade econômica. 
Diante do exposto, este estudo tem como objetivo geral analisar a trajetória recente ocupação e renda das mulheres no mercado de trabalho nordestino. A metodologia empregada consiste em uma análise estatística dos dados extraídos do Programa Nacional por Amostra Domiciliar (PNAD) do Instituto Brasileiro de Geografia e Estatística (IBGE) considerando o período de 2001 a 2015.

As próximas seções estão estruturadas da seguinte maneira: a seção 2 faz uma revisão da trajetória da inserção feminina no mercado de trabalho apresentando ainda algumas características da mão de obra feminina no Nordeste, a seção 3 apresenta os procedimentos metodológicos, a seção 4 apresenta os resultados e em seguida são apresentadas as considerações finais.

\section{REFERENCIAL TEÓRICO}

\subsection{TRAJETÓRIAS DA MULHER NO MERCADO DE TRABALHO}

São vastos os números de pesquisas que investigam a progressiva participação da mulher no mercado de trabalho e o aumento da sua importância econômica, bem como sua responsabilidade em melhorar as condições financeiras da família e o seu destaque profissional em diversos setores. Cresce também o número de mulheres em postos diretivos nas empresas. Tal evolução se dá em vários países de maneira semelhante (ZILLI, 2004).

Kon (2013) expõe que a ampliação da participação feminina no mercado de trabalho se dá em grande medida pela difusão do processo de globalização. $O$ processo de progresso tecnológico das últimas décadas impulsionou o surgimento de novas atividades, bem como na transformação de atividades já existentes. Esse cenário foi possibilitando um contingente cada vez maior de mulheres participando da produção econômica.

Para Flecha (2007) a ampliação da inserção feminina no trabalho foi impulsionada em boa parte pelos movimentos feministas e da organização das mulheres em reivindicar igualdade de condições de trabalho. Assim ao longo do tempo a organização feminina em termos dos movimentos sociais possibilitaram o crescimento da inserção da mulher no mercado de trabalho.

Probst e Ramos (2003) explicam que o crescimento do volume de mulheres empregadas ganhou força com a criação de leis e marcos institucionais que 
flexibilizaram relações de trabalho, o que possibilitou maior proteção ao trabalho feminino, redução de carga horária e melhores condições de trabalho. No Brasil, por exemplo, isso pode ser constatado pela constituição de 1932.

Diante desse processo flexibilização legal e de crescimento dos movimentos sociais e feministas, as mulheres ingressam no mercado de trabalho também devido a um contexto de expansão da economia com acelerado processo de industrialização e urbanização que vinha-se sofrendo. A década de 1980 deu sequência ao processo, apesar da estagnação da atividade econômica no nosso país. A abertura econômica ocorrida na década de 1990 trouxe junto com algumas multinacionais, o conceito da força de trabalho feminino, da importância da mulher como consumidora e como formadora de opinião e como mão-de-obra diferenciada. (BRUSCHINI; LOMBARDI, 1999).

Cabe frisar que trabalho doméstico e os cuidados com os filhos ainda são fatores importantes a considerar quando se trata do tema. Segundo Santana (2006), o trabalho doméstico é um trabalho não produtivo, ou seja, não produz valor. Em razão disto, acaba como sendo não reconhecido os esforços da mulher perante a família e a sociedade no geral. Face aos desdobramentos da economia moderna, as mulheres acabam por migrar para o trabalho formal, porém na maioria das vezes não ocorre a troca das funções, e sim o acúmulo das duas atividades.

Nos últimos anos, consegue-se observar que a participação das mulheres no mercado de trabalho brasileiro cresceu consideravelmente. Porém, mesmo frente a um crescimento significativo da força de trabalho feminina ocupada, ainda persistem diferenças expressivas em termos de inserção e remuneração. Na segregação ocupacional, mesmo que os homens e as mulheres tenham capacidade igualitária em assumir a mesma função, as chances de homens ocuparem empregos de maior remuneração, são maiores do que as das mulheres (FOUARGE; KRIECHEL; DOHMEN, 2014).

No Brasil, as estatísticas de gênero da Pnad contínua (2018) apresentam informações relevantes na avaliação do processo de inserção da mulher no mercado de trabalho. Em termos do tempo dedicado aos afazeres domésticos e/ou cuidados com outras pessoas, as mulheres dedicam 18,1 horas frente a 10,5 dos homens. Se considerar a região nordeste as mulheres dedicam 19 horas enquanto os homens 
10,5. Isso pode explicar o fato de que a taxa de inserção masculina no mercado de trabalho significativamente superior a feminina.

A carga horária de trabalho contribui da mesma forma para os diferenciais de inserção. No Brasil, os dados da Pnad contínua (2018) mostram um percentual muito mais elevado de mulheres ocupadas tem tempo parcial $(28,2 \%)$ do que os homens (14,1\%). Na região Nordeste esse percentual é ainda mais expressivo, sendo que as mulheres ocupadas em tempo parcial representam $36,9 \%$ e os homens $22,3 \%$.

Outro ponto bastante discutido é o diferencial de salários entre homens e mulheres. Nesse aspecto, os dados da Pnad evidenciam que os rendimentos médios das mulheres são $3 / 4$ dos rendimentos médios dos homens. Um dos fatores que podem influenciar esse diferencial é o tipo de ocupações que as mulheres se inserem em relação aos homens, sobretudo, postos de trabalho em tempo parcial.

Colocado esses pontos, a próxima seção faz uma análise das principais características da mão de obra feminina no Nordeste, considerando contingente, ocupação e escolaridade.

\subsection{ESTRUTURA OCUPACIONAL DA MÃO-DE-OBRA FEMININA NO NORDESTE}

Esta seção tem como objetivo discutir os aspectos mais gerais da estrutura da mão de obra feminina no Nordeste. Apresenta a distribuição da mão de obra feminina em termos de condição da ocupação, setores da atividade e escolaridade.

De acordo com a Pesquisa Nacional por Amostra de Domicílios (PNAD, 2015), apesar das mulheres representarem a maioria relativa demográfica $(51,5 \%)$ apenas $54,4 \%$ delas estavam em situação de ocupação, em contraste com os $76,2 \%$ dos homens.

De acordo com a tabela 1, pode-se observar que na região do Nordeste brasileiro no período de 2001 a 2015, não houve expressivos aumentos no número de mulheres ocupadas. Isso pode ser inferido a partir da observação que em praticamente todos os estados os percentuais de mulheres ocupadas são crescentes ao longo da série. 
Tabela 1 - Percentual de mulheres de 10 anos ou mais de idade ocupadas na semana de referência (Mil pessoas) na região Nordeste.

\begin{tabular}{c|ccccccccccccccc}
\hline \multicolumn{1}{c}{$\mathbf{2 0 0 1}$} & $\mathbf{2 0 0 2}$ & $\mathbf{2 0 0 3}$ & $\mathbf{2 0 0 4}$ & $\mathbf{2 0 0 5}$ & $\mathbf{2 0 0 6}$ & $\mathbf{2 0 0 7}$ & $\mathbf{2 0 0 8}$ & $\mathbf{2 0 0 9}$ & $\mathbf{2 0 1 1}$ & $\mathbf{2 0 1 2}$ & $\mathbf{2 0 1 3}$ & $\mathbf{2 0 1 4}$ & $\mathbf{2 0 1 5}$ \\
\hline MA & 39,3 & 39,5 & 39,8 & 42,5 & 40,9 & 41,2 & 41,9 & 39,4 & 38,6 & 39,2 & 40,3 & 40,8 & 42,4 & 39,5 \\
PI & 39,4 & 40,5 & 43,8 & 41,7 & 43,1 & 44,2 & 43,1 & 44,0 & 44,0 & 42,4 & 42,0 & 42,6 & 43,2 & 43,8 \\
CE & 42,4 & 41,4 & 40,6 & 42,1 & 42,9 & 41,8 & 42,0 & 43,4 & 42,9 & 41,6 & 41,0 & 42,4 & 41,6 & 41,5 \\
RN & 39,5 & 37,5 & 38,1 & 40,1 & 39,5 & 39,7 & 41,1 & 40,5 & 39,4 & 39,5 & 40,4 & 39,8 & 41,1 & 41,1 \\
PB & 37,4 & 38,5 & 39,5 & 39,0 & 41,1 & 40,5 & 39,6 & 38,7 & 37,7 & 40,6 & 42,0 & 40,6 & 42,1 & 42,2 \\
PE & 40,5 & 40,8 & 40,2 & 40,3 & 41,0 & 41,2 & 39,8 & 41,0 & 39,4 & 39,0 & 40,5 & 40,3 & 41,6 & 41,1 \\
AL & 39,3 & 38,0 & 37,9 & 37,7 & 39,9 & 40,1 & 39,5 & 38,9 & 38,5 & 36,5 & 38,1 & 39,5 & 37,8 & 37,9 \\
SE & 40,3 & 41,9 & 43,6 & 42,2 & 42,1 & 42,4 & 44,3 & 43,2 & 42,6 & 39,5 & 41,7 & 39,3 & 43,6 & 42,3 \\
BA & 38,8 & 40,0 & 39,3 & 39,6 & 40,4 & 40,8 & 40,6 & 41,5 & 41,7 & 41,8 & 40,8 & 41,5 & 42,6 & 42,3 \\
\hline
\end{tabular}

Estas informações comprovam boa parte da literatura acerca da ampliação da oferta de trabalho feminina. Barbosa (2014) estima os determinantes da participação feminina utilizando características individuais das mulheres. Para isso o autor identifica os fatores que determinam a entrada das mulheres no mercado de trabalho brasileiro. Os resultados encontrados evidenciaram que os anos de estudo são um fator significativo que tem permitido a ampliação da participação feminina no mercado de trabalho.

Dentro desta mesma perspectiva Resende (2016) pesquisou a trajetória da taxa de participação da mulher no mercado de trabalho brasileiro, investigando também seus determinantes. Mostrou que a ampliação da participação feminina se dá em função em boa medida de sua escolaridade.

O gráfico 1, representa a taxa de crescimento por agrupamento de atividades das pessoas de 10 anos de idade ou mais ocupadas na semana de referência na região Nordeste, conforme dados do Instituto Brasileiro de Geografia e Estatística (IBGE), por meio do Sistema IBGE de Recuperação Automática (SIDRA): 
Gráfico 1 - Percentual de mulheres de 10 anos ou mais de idade ocupadas na semana de referência (por grupamento de atividade) - percentual do total geral, Nordeste.

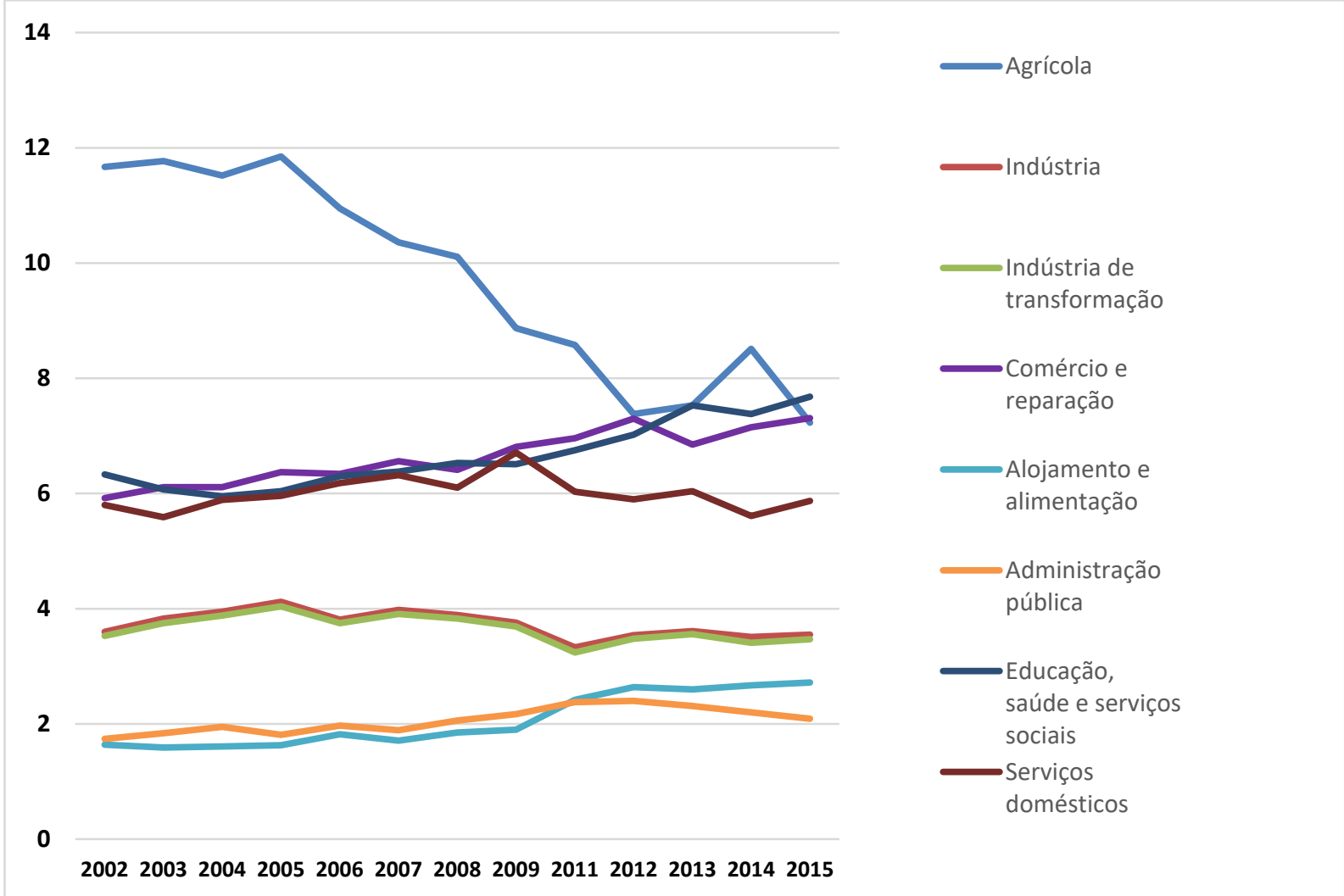

Fonte: IBGE/SIDRA.

A atividade agrícola foi o que mais caiu no período de 2005 a 2015, enquanto os demais setores mantiveram-se constantes ou em situação de oscilação. A atividade de educação, saúde e serviços sociais, e a atividade de comércio e reparação foram as que mais estiveram em elevação no período. Isso pode ainda ser o resultado da melhor formação escolar da mulher ao longo do tempo, bem como do processo de crescimento dos setores ligados a serviços que demandam boa parte da mão de obra feminina.

Conforme o levantamento realizado pelo Serviço Brasileiro de Apoio às Micro e Pequenas Empresas (SEBRAE,2018), no ano de 2016 nas pequenas empresas, as mulheres eram parte de $53 \%$ da mão de obra empregada, número maior que os homens que atuam no setor, com 47\%. Este mesmo estudo mostrou que houve redução nas disparidades de remuneração entre os sexos no período de 2006 a 2016.

Na região Nordeste, nos pequenos negócios concentra-se 59,2\% da mão de obra feminina empregada regularmente formalizada. Ficando a região em penúltimo lugar dentre as que mais empregam formalmente mulheres neste setor de atividade. 
Perdendo apenas para o Sudeste com $56,1 \%$. As demais regiões ficaram o CentroOeste com $62,3 \%$, seguido da região Sul com $62,2 \%$ e da região Norte com $60,3 \%$. Em 2016, a média nacional apontava que 58,4\% do emprego formal nos pequenos negócios eram compostos por mulheres, enquanto $41,6 \%$ estavam ocupando funções nas médias e grandes empresas. Os homens por sua vez, ocuparam 51,9\% das funções nas pequenas empresas.

A mesma pesquisa apontou que as disparidades nos rendimentos médios femininos em comparação com os masculinos tiveram uma queda de quase $4 \%$. No período de 2006 a 2016, as diferenças salariais caíram de 20\% para 16,8\%. O aumento da participação feminina no setor de serviços, em específico nas microempresas, começou a ganhar destaque a partir de 2009, quando ultrapassaram $51 \%$ da ocupação nas funções do setor. Já no comércio, $55 \%$ das funções são ocupadas por homens, em contraposição a $45 \%$ ocupadas por mulheres.

O gráfico 2 apresenta o percentual de mulheres ocupadas por anos de estudo, de acordo com dados da Pnad, conforme pode-se observar a seguir:

Gráfico 2: Percentual de mulheres de 10 anos ou mais ocupadas por anos de estudo - Nordeste.

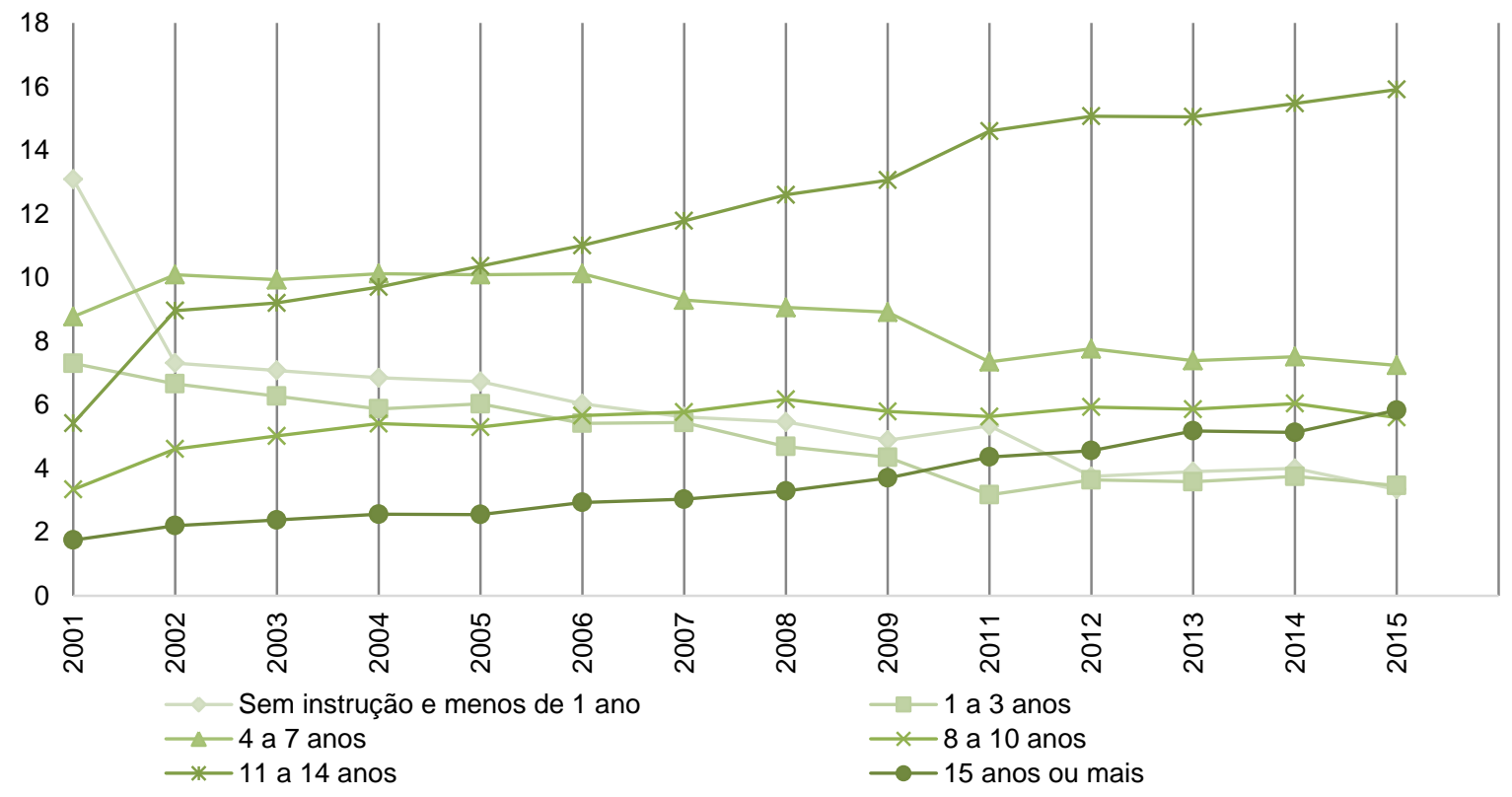

Fonte: IBGE/PNAD.

De acordo com os dados apresentados no gráfico 2, observa-se um fenômeno muito importante na região nordeste, que é o crescimento das mulheres 
com escolaridade na faixa de 11 a 14 anos de estudo, bem como a faixa de 15 anos ou mais. Ao mesmo tempo em que os percentuais das mulheres sem instrução e na faixa de 4 a 7 anos de estudo tem reduzido ao longo da série estudada.

Isso pode explicar em boa medida uma ampliação da participação feminina no mercado de trabalho, tanto em termos empregos com maiores remunerações e com melhores condições de trabalho. Parte expressiva da literatura acerca dos determinantes da participação feminina no mercado de trabalho e mesmo da oferta de horas de trabalho das mulheres, têm atribuído a ampliação da escolaridade um fator preponderante na probabilidade de participação e na decisão de alocação de horas.

Feitas essas considerações acerca dos aspectos mais gerais do mercado de trabalho do Nordeste, a próxima seção apresenta a estratégia metodológica adotada.

\section{METODOLOGIA}

Inicialmente foi utilizada como amostra, a população feminina economicamente ativa e ocupada dos bancos de dados dos órgãos oficiais de estatística de emprego e desemprego referente à região Nordeste.

Os dados estatísticos foram obtidos junto aos órgãos oficiais de estatística: Instituto Brasileiro de Geografia e estatística (IBGE), Sistema de Recuperação de Dados (SIDRA). O banco de dados utilizado será composto basicamente pelo quantitativo de mulheres ocupadas e das características de rendimento dos domicílios.

Uma vez preparado o banco de dados, estes foram tabulados e analisados a partir da estatística descritiva, observando a trajetória da estrutura ocupacional e da renda das mulheres ocupadas na região Nordeste.

\section{RESULTADOS E DISCUSSÃO}

No Brasil, o aumento da participação da mulher no mercado de trabalho torna-se cada vez mais notória principalmente com a expansão da economia. Entretanto, as mulheres brasileiras ainda recebem em média $70 \%$ do 
salário, mas ainda se encontram em defasagem considerável quando comparados com o salário dos homens.

No Brasil, conforme dados da Pnad, as famílias chefiadas por mulheres representam 24,9\% dos domicílios brasileiros. O Nordeste é a região brasileira que apresenta a maior proporção de domicílios chefiados por mulheres, com 25,9\%, acompanhado da região sudeste com $25,6 \%$.

Tabela 2: Famílias residentes em domicílios particulares chefiadas por mulheres por região

\begin{tabular}{lrrrrrrrr}
\hline & $\mathbf{2 0 0 1}$ & $\mathbf{2 0 0 3}$ & $\mathbf{2 0 0 5}$ & $\mathbf{2 0 0 7}$ & $\mathbf{2 0 0 9}$ & $\mathbf{2 0 1 1}$ & $\mathbf{2 0 1 3}$ & $\mathbf{2 0 1 5}$ \\
\hline Norte & 29,11 & 32,84 & 29,02 & 34,23 & 34,08 & 37 & 38,68 & 39,42 \\
\hline Nordeste & 27,79 & 29,34 & 31,33 & 33,42 & 34,83 & 38,64 & 40,06 & 42,94 \\
\hline Sudeste & 28,02 & 29,24 & 31,43 & 33,18 & 35,99 & 37,55 & 38,65 & 40,67 \\
\hline Sul & 24,42 & 26,37 & 27,56 & 30,86 & 32,94 & 36,29 & 37,51 & 36,87 \\
\hline C. Oeste & 26,66 & 26,3 & 30,29 & 32,61 & 36,14 & 35,46 & 38,62 & 39,5 \\
\hline
\end{tabular}

Fonte: PNAD/SIDRA.

A partir da tabela 2, observa-se que foi na região Nordeste que se concentrou o maior número de famílias chefiadas por mulheres. Isto se deve ao fato de que a maior autonomia destas mulheres refletiu sobre as relações sociais, e com isto, houve uma quebra de padrões existentes onde o homem deve ser obrigatoriamente o chefe da família, cabendo a mulher apenas cuidar da casa e dos filhos.

O número de famílias chefiada por mulheres teve um aumento de $26,5 \%$ registrado no ano de 2004 para 38,8\% no ano de 2014. Quanto as mulheres conjugadas chefiaram 3,5\% dos domicílios em 2004 para em 2014 com 13,5\%. Isto representa um avanço no que diz respeito aos direitos sociais, histórico e culturalmente negados às mulheres, que vem sendo adquiridos através da quebra de paradigmas e padrões, fazendo com isto que as mulheres possuam cada vez mais protagonismo perante a sociedade moderna, e com isto autonomia semelhante a autonomia masculina.

lasi (1991, p. 01) argumenta que a contemporaneidade trouxe consigo uma noção de igualdade formal entre homens e mulheres, no que tange aos salários, direitos sindicais e participação política. Isso pode deixar transparecer a ilusão de que a igualdade formal também se desenvolve de forma material.

As mulheres correspondem a pouco mais da metade da população nacional e, ainda assim, o desemprego entre os homens é de 10,6\% enquanto, entre as 
mulheres, o índice é de 13,8\%, ao mesmo tempo que atualmente, as mulheres são responsáveis pelo sustento de $37,3 \%$ das famílias.

Dados da última Pnad divulgada mostra que viviam no Brasil 103,5 milhões de mulheres, o correspondente a $51,4 \%$ da população. Além disso, as mulheres ocupam apenas $37 \%$ dos cargos de liderança das empresas brasileiras, sendo que esses números caem para $10 \%$ em grandes empresas. Quando conseguem alcançar cargos de gerência e direção dentro das corporações, as mulheres recebem, cerca de, $46.7 \%$ a menos do que os homens.

Gráfico 3: Distribuição das mulheres por condição da ocupação, Nordeste - 2001 a 2015.

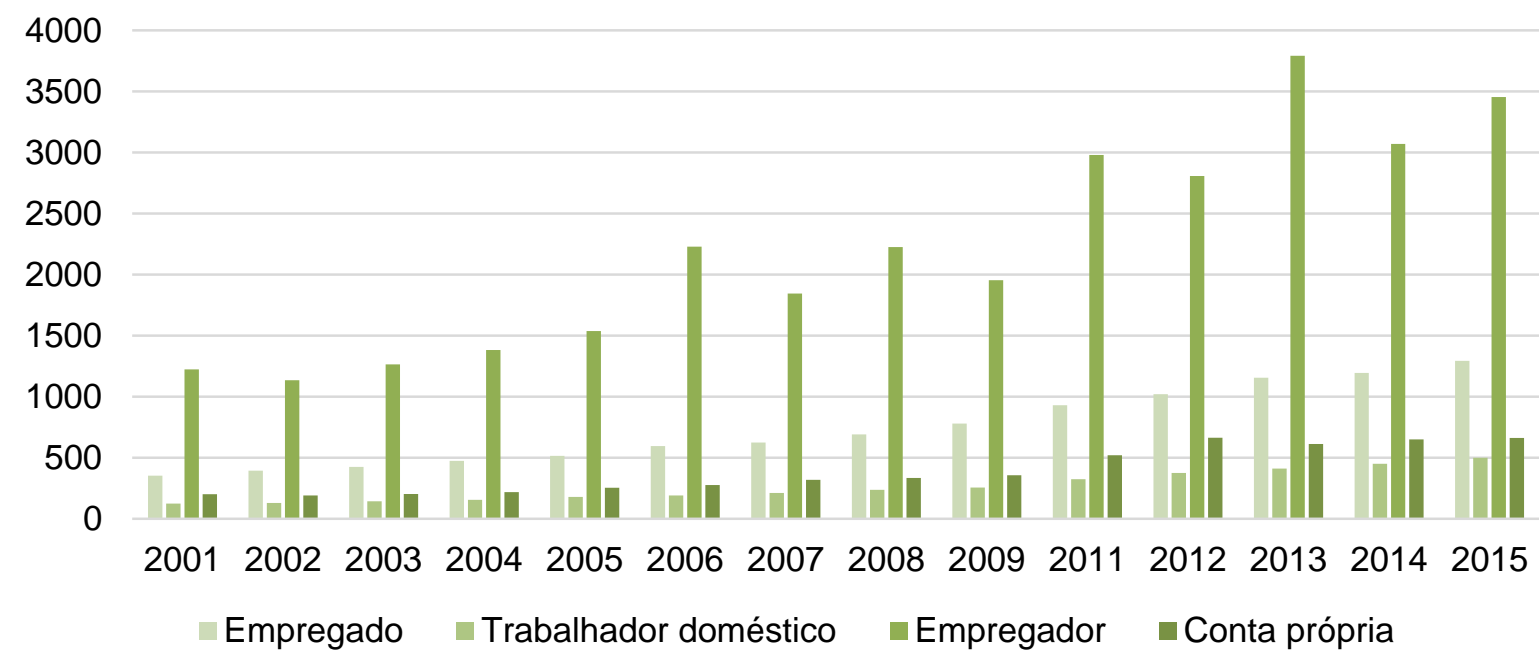

Fonte: IBGE/SIDRA.

A partir desses dados, fica evidente o crescimento das mulheres em basicamente todos os tipos de condição da ocupação, com destaque para as mulheres na condição de empregada e empregadora. Este resultado pode se dar sobretudo em face do crescimento da escolaridade feminina que permite as mulheres empreender e da mesma forma de se inserir melhor no mercado de trabalho.

Os dados da Pnad de 2014 mostram que as mulheres contribuem com 40,9\% para a renda familiar; entre os homens, essa contribuição é de 59,1\%. Já as mulheres de áreas rurais, tem a participação no rendimento um pouco maior de $42,4 \%$. A contribuição das mulheres é mais importante na zona rural nordestina (51\%). E é menor na zona rural do Centro-Oeste (26\%). Esses dados passam por mudanças quando as mulheres consideradas brancas contribuem com uma parcela 
de $39,7 \%$ na renda familiar. Se o chefe da família era negro ou pardo, a contribuição da mulher era maior que $42 \%$.

Nas famílias formadas por casais com filhos, a participação feminina foi menor que $31,7 \%$, enquanto nas famílias monoparentais essa participação foi de $70,8 \%$, quando elas eram responsáveis pelo sustento. Ainda foi mostrado que na grande parte do nordeste a contribuição feminina para o rendimento familiar era maior do que a dos homens.

Em termos de faixa de rendimento, o gráfico 4 apresenta a distribuição percentual das mulheres por faixa de renda média mensal. Os dados apontam que embora a taxa de crescimento da participação feminina tenha sido significativa como apontam algumas evidências já explicadas acima, o maior percentual de mulheres ainda está concentrado em faixas mais baixas de renda.

No entanto, observa-se um crescimento das mulheres com rendimento de mais de meio até um salário mínimo e com mais de uns até dois salários mínimos, frente as mulheres como rendimento meio salário mínimo. Isto evidencia a importância da educação e qualificação da mulher na obtenção de salários com melhores rendimentos.

Gráfico 4 - Mulheres de 10 anos ou mais de idade ocupadas e Valor do rendimento médio mensal, Nordeste.

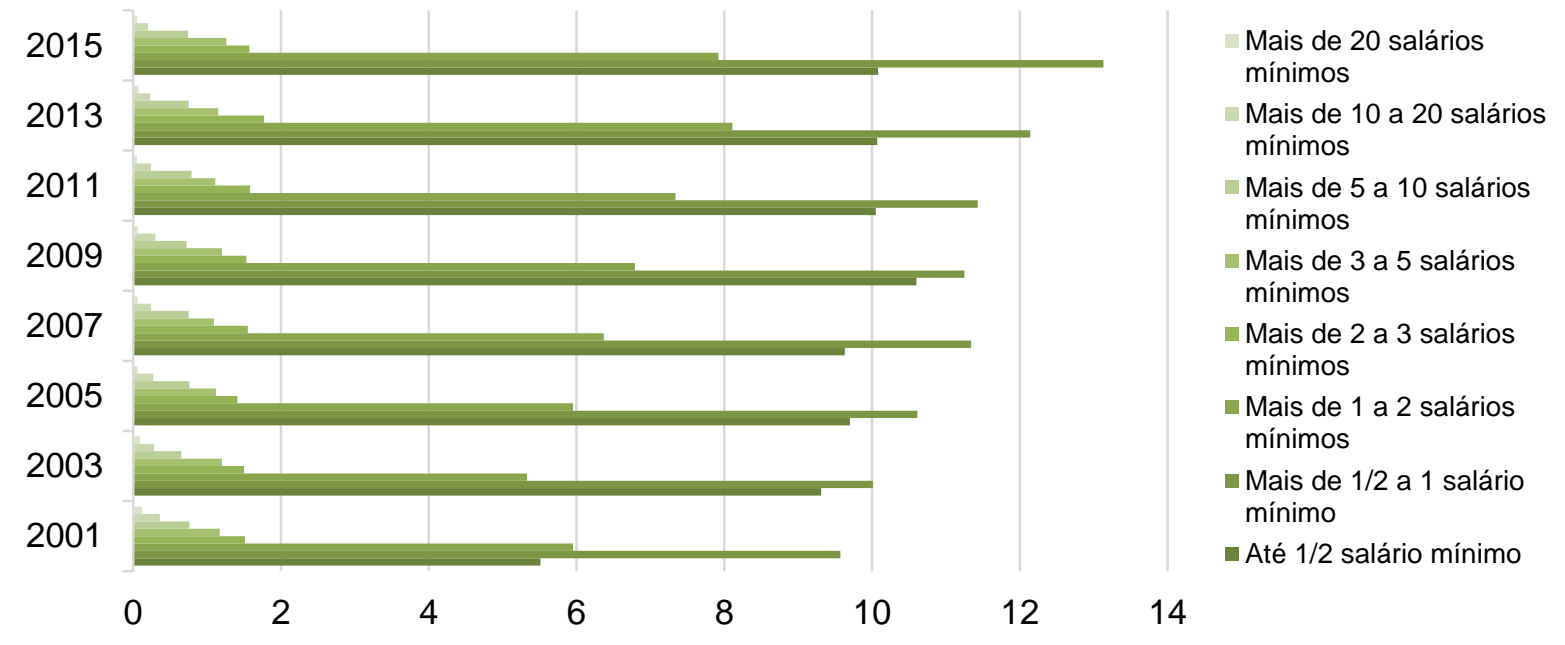

Fonte: PNAD/SIDRA.

Algumas conclusões que os dados acerca dos rendimentos médios das mulheres apresentados permitem inferir, estão relacionadas a dificuldade que ainda existe em equalizar a questão salarial de homens e mulheres. Embora esta pesquisa 
não se dedicou a esse tema, algumas pesquisas mais focalizadas nessa perspectiva argumentam que ainda existe um forte diferencial de rendimento por gênero no mercado de trabalho.

Outro ponto importante é avaliar as faixas de rendimento por grupamento de atividade. Essas informações são apresentadas na tabela 03. Os dados permitem observar uma queda da participação das mulheres ocupadas no setor agrícola com rendimento de até meio salário mínimo, o que pode estar evidenciando uma migração das mulheres para setores que oferecem melhores salários e com melhores condições de trabalho.

Tabela 03: Percentual de mulheres de 10 anos ou mais de idade ocupadas na semana de referência por faixa de rendimento e grupo de atividades (Mil pessoas), Região Nordeste 2002 e 2015.

\begin{tabular}{|c|c|c|c|c|c|c|}
\hline & \multicolumn{6}{|c|}{2002} \\
\hline & Até $1 / 2$ & $\begin{array}{c}\text { Mais } \\
\text { de } 1 / 2 \\
\text { a } 1\end{array}$ & $\begin{array}{c}\text { Mais de } \\
1 \mathrm{a} 2\end{array}$ & $\begin{array}{c}\text { Mais de } \\
2 \text { a } 5\end{array}$ & $\begin{array}{c}\text { Mais de } \\
5 \text { a } 10\end{array}$ & $\begin{array}{l}\text { Mais } \\
\text { de } 10\end{array}$ \\
\hline Agrícola & 1,63 & 0,57 & 0,11 & 0,03 & 0 & 0 \\
\hline Indústria & 1,29 & 0,92 & 0,74 & 0,2 & 0,05 & 0,03 \\
\hline Indústria de transformação & 1,29 & 0,92 & 0,72 & 0,19 & 0,04 & 0,03 \\
\hline Comércio e reparação & 1,53 & 1,45 & 1,35 & 0,58 & 0,13 & 0,03 \\
\hline Alojamento e alimentação & 0,38 & 0,53 & 0,35 & 0,11 & 0,01 & 0,01 \\
\hline Administração pública & 0,07 & 0,68 & 0,4 & 0,3 & 0,17 & 0,12 \\
\hline \multirow[t]{3}{*}{ Educação, saúde e serviços sociais } & 0,45 & 1,97 & 2,13 & 1,29 & 0,29 & 0,13 \\
\hline & \multicolumn{6}{|c|}{2015} \\
\hline & Até $1 / 2$ & $\begin{array}{c}\text { Mais } \\
\text { de } 1 / 2 \\
\text { a } 1\end{array}$ & $\begin{array}{c}\text { Mais de } \\
1 \mathrm{a} 2\end{array}$ & $\begin{array}{c}\text { Mais de } \\
2 \text { a } 5\end{array}$ & $\begin{array}{c}\text { Mais de } \\
5 \text { a } 10\end{array}$ & $\begin{array}{l}\text { Mais } \\
\text { de } 10\end{array}$ \\
\hline Agrícola & 1,4 & 0,31 & 0,08 & 0,02 & 0,01 & - \\
\hline Indústria & 1,14 & 1,2 & 0,76 & 0,21 & 0,05 & 0,02 \\
\hline Indústria de transformação & 1,13 & 1,19 & 0,74 & 0,17 & 0,04 & 0,01 \\
\hline Comércio e reparação & 1,47 & 2,55 & 2,16 & 0,5 & 0,1 & 0,03 \\
\hline Alojamento e alimentação & 0,53 & 1,1 & 0,68 & 0,14 & 0,02 & 0 \\
\hline Administração pública & 0,06 & 0,78 & 0,53 & 0,43 & 0,2 & 0,07 \\
\hline Educação, saúde e serviços sociais & 0,35 & 2,37 & 2,58 & 1,87 & 0,35 & 0,11 \\
\hline
\end{tabular}

Fonte: IBGE/SIDRA.

Quando se observa os demais setores, nota-se que ao se comparar o ano de 2002 e o ano de 2015, as faixas de rendimento acima de um salário mínimo 
apresentam relativo crescimento na comparação realizada, podendo dar destaque aos setores ligados ao comercio, serviços e administração pública.

De forma geral, o panorama dos dados mostra que as mulheres vêm melhorando sua condição de renda do emprego, bem como da melhoria das condições de trabalho dada a ampliação da participação em setores menos insalubres.

Assim, a análise dos dados e informações acerca da participação e da renda da mão de obra feminina na região Nordeste, mostra que as mulheres têm ocupado cada vez mais espaços dentro do mercado de trabalho o que pode ser explicado pela melhoria da qualificação e da estrutura dos domicílios.

Observou-se ainda que a renda da mão de obra feminina também vem se elevando no período analisado, sobretudo por faixa de escolaridade e por grupamento das atividades. No entanto essa melhoria da renda ainda se encontra em um contexto de significativo diferencial de rendimentos entre gênero e requer da mesma forma políticas públicas que melhorem o acesso da mulher no mercado de trabalho.

\section{CONSIDERAÇÕES FINAIS}

O objetivo deste estudo foi analisar a trajetória da renda das mulheres ocupadas na região nordeste, para isso, a metodologia empregada foi a partir de dados extraídos do Programa nacional por Amostra Domiciliar (PNAD) do Instituto Brasileiro de Geografia e Estatística (IBGE).

Em uma análise da trajetória da participação feminina no mercado de trabalho, observa-se que as mulheres vêm ampliando sua participação no mercado de trabalho, como resultado de um processo socioeconômico inserido na quebra de padrões sociais e no crescimento do progresso tecnológico e econômico.

A estrutura da ocupação feminina no Nordeste mostrou nuances interessantes: o setor agrícola foi o ramo que com o passar dos anos menos empregou mulheres, por outro lado as atividades de educação, saúde e serviços sociais, junto com o setor de comercio e reparação, foram cada vez mais empregando mulheres. $59,2 \%$ da mão de obra feminina formal encontra-se nos 
pequenos negócios, observou-se ainda que no período de 2001 a 2015 aumentou o número de mulheres com escolaridade de 11 a 14 anos de estudo.

Verificando o perfil da renda feminina no Nordeste mediante características individuais e do domicílio notou-se que houve um aumento de 25,9\% de domicílios chefiados por mulheres. Na zona rural nordestina, a contribuição das mulheres no orçamento familiar chega a $51 \%$.

No período de análise, o rendimento das mulheres aumentou significativamente na faixa de renda entre meio salário a um salário mínimo nacional, evidenciando uma melhoria em algumas faixas de remuneração, embora os diferenciais de salários frente aos homens ainda persistem. Disso conclui-se que na região Nordeste, assim como no Brasil, as mulheres têm ganhado espaço no mercado de trabalho, embora se percebam algumas distorções em relação aos homens, sobretudo, no que tange a inserção e remuneração.

\section{REFERÊNCIAS}

BARBOSA, Ana Luiza Neves de Holanda. Participação feminina no mercado de trabalho brasileiro. Nota Técnica: Mercado de Trabalho 31- 41. IPEA, 2014.

BRUSCHINI, Cristina; LOMBARDI, Maria Rosa. A bipolaridade do trabalho feminino no Brasil contemporâneo. Cadernos de pesquisa, n. 110, p. 67-104, 2000.

CIRINO, Jader Fernandes; DE LIMA, João Eustáquio. Determinantes da participação feminina no mercado de trabalho: uma comparação entre os sexos e entre os mercados das regiões metropolitanas de Belo Horizonte e Salvador. Revista Econômica do Nordeste, v. 42, n. 1, p. 165-182, 2011.

FLECHA, Marilia Mariano de Lima. A Inserção da Mulher no Mercado de Trabalho: Um Retrato da Desigualdade na Região Metropolitana de Belo Horizonte. XXXI Encontro da ANPAD, Rio de Janeiro, 2007.

FOUARGE, D., KRIECHEL, B., e DOHMEN, T. Occupational sorting of school graduates: The role of economic preferences. Journal Of Economic Behavior \& Organization, 2014.

KON, Anita. Assimetrias entre gêneros no mercado de trabalho brasileiro: crise e políticas públicas. Revista Ciências do Trabalho, v. 1, n. 1, p. 113-140, 2013.

LEONE, Eugenia Troncoso; TEIXEIRA, Marilane Oliveira. As mulheres no mercado de trabalho e na organização sindical. Anais do XVII Encontro Nacional de

Estudos Populacionais p. 1-21, 2016. 
IBGE. Pesquisa nacional por amostra de domicílios contínua. Disponível em: https://www.ibge.gov.br/estatisticas/sociais/populacao/9171-pesquisa-nacional-por-amostra-dedomicilios-continua-mensal.html?=\&t=o-que-e Acesso em novembro de 2019.

IASI, M. L. Olhar o mundo com olhos de mulher. Instituto Internacional de Investigação e Formação. Amsterdã, Holanda, 1991.

PROBST, Elisiana Renata; RAMOS, Paulo. A evolução da mulher no mercado de trabalho. Santa Catarina: Instituto Catarinense de Pós-Graduação, p. 1-8, 2003.

RAMOS, L; AGUAS, M. F. F; FURTADO, L. M. S. Participação Feminina na Força de Trabalho do status Socioeconômico das Famílias, Economia Aplicada, V. 15, n. 4, 2011 , pp. $595-611$.

RESENDE, Ana Carolina Marinato. A evolução da taxa de participação feminina no mercado de trabalho brasileiro nos últimos 20 anos. Trabalho de Conclusão de Curso (Bacharelado em Economia) Pontifícia Universidade Católica do Rio de Janeiro. Rio de Janeiro, 2016.

SANTANA, Mônica Cristina Silva. Muito Trabalho, Pouco Poder: Participação Feminina Mitigada Nos Assentamentos Rurais do Estado de Sergipe. In: GROSSI, Miriam Pilar. ; SCHWADE, Elisete. (Org.) Política e Cotidiano: estudos antropológicos sobre gênero, família e sexualidade. Blumenau: Nova Letra. p. 47 $-68,2006$.

SIDRA, I. B. G. E. Sistema IBGE de recuperação automática. Rio de Janeiro. Disponível em : https://sidra.ibge.gov.br/pesquisa/pnad/geral/pesquisa-basica Acesso em: Novembro, 2019.

SEBRAE, Serviço Brasileiro de Apoio às Micro e Pequenas Empresas; Departamento Intersindical de Estatística e Estudos Socioeconômicos DIEESE. Anuário do trabalho nos pequenos negócios: 2016. 9.ed São Paulo- SP 2018

ZILLI, S. M. Mujer, discriminación y derecho del trabajo. Revista Observatório Social, n. 5, 2004. 